\title{
A Case of an Infectious Abdominal Aortic Aneurysm Presenting Twelve Years after an Acute Q-Fever Infection
}

\author{
Pierre-Olivier Lebel ${ }^{1}$, Stefan Luu ${ }^{1}$, Mathieu Pelletier ${ }^{1 *}$, Nicolas Aubree ${ }^{2}$, Jean-Francois Boisvert ${ }^{3}$ \\ ${ }^{1}$ Unité de Médecine Familiale du Nord de Lanaudière, Université Laval, St-Charles-Borromée, Qc, Canada \\ ${ }^{2}$ Department of Surgery, Centre Hospitalier Régional de Lanaudière, St-Charles-Borromée, Qc, Canada \\ ${ }^{3}$ Department of Medicine, Centre Hospitalier Régional de Lanaudière, St-Charles-Borromée, Qc, Canada
}

Received: May 25, 2015; Accepted: August 04, 2015; Published: August 26, 2015

*Corresponding author: Mathieu Pelletier, Unité de Médecine Familiale du Nord de Lanaudière, Université Laval, St-Charles-Borromée, Qc, Canada, Email: math.pelletier@videotron.ca

\begin{abstract}
Introduction: $\mathrm{Q}$ fever is a zoonotic disease caused by Coxiella burnetii. These are acute and chronic infections, and possible clinical manifestations are varied and non-specific. Diagnosis, follow up and treatment can be challenging. A high level of suspicion is crucial to prevent complications.

Case report: A 70-year-old man, who was treated 12 years beforehand for an acute $\mathrm{Q}$ fever, was admitted for abdominal pain and chills. A computed tomography angioscan revealed an ulceration of an aortic aneurysm. He also had a psoas collection containing necrotizing granulomatous inflammation revealed by a CT-guided aspiration. Surgical resection of the ulcerated aneurysm as well as an aorto-aortic bypass with Dacron graft was performed. PCR on the mycotic aneurysm confirmed a chronic $\mathrm{Q}$ fever infection. A long-term treatment with doxycycline and hydroxychloroquine is ongoing and will likely be required for life.

Discussion: After an acute infection, patients should undergo close serological follow-up. Approximately $1.5-11 \%$ of patients with acute $\mathrm{Q}$ fever go on to develop Chronic Q- fever. Recent data from Dutch epidemic have shown that chronic vascular Q-fever infection is more usual than we thought compared to endocarditis. Significant host risk factors now include valvular surgery, vascular and cardiac prosthesis, aneurysms and renal insufficiency in age $>60$ years old. Experts recommend surgical resection and long-term antibiotics for the treatment of chronic vascular infections.

Conclusion: This case of chronic vascular $Q$ fever is particularly due to the impressively long delay between the acute infection and vascular complication (12 years). The case also raises interesting discussion topics on physiopathology and histopathological diagnosis of vascular Q-Fever infection. It is of mention to know that the patient did not have any aneurysm on investigation performed during acute Q-Fever.
\end{abstract}

Keywords: Aortic; Aneurysm; Q-fever; Chronic; Infection

\section{Introduction}

Q fever is a zoonotic disease caused by the proteobacteria Coxiella burnetii. They are acute and chronic infections. Clinical manifestations are varied and non-specific. Diagnosis, follow up and treatment can be challenging and a high level of suspicion is crucial to prevent complications. We report a case of chronic Q fever presenting with an abdominal aortic aneurysm twelve years after an acute infection.

\section{Case-Report}

\section{Acute infection}

In September 1998, a 58-year-old man presented with fever and night sweats since few weeks. He had returned from a vacation in the Gaspesiae region (Canada). He had not been in the wilderness nor he visited a farm. He visited a disused mine. Physical exam showed left upper quadrant sensitivity. Liver function tests (LFTs) were abnormal: ALT $144 \mathrm{U} / \mathrm{L}$ (reference 0-55 U/L), PALC $156 \mathrm{U} / \mathrm{L}$ (reference 40-150 U/L) and GGT $204 \mathrm{U} / \mathrm{L}$ (reference 12-64 U/L). C-reactive protein was 48 $\mathrm{mg} / \mathrm{L}$ (reference $<8 \mathrm{mg} / \mathrm{L}$ ), whereas lung x-rays, blood and urine cultures were negative. A trans-thoracic echocardiogram failed to demonstrate any signs of endocarditis. Abdominal CT-scan showed hepatic steatosis. The aorta had moderate atheromatosis but no aneurysm demonstrated. Endoscopic retrograde cholangiopancreatography, Gallium scintigraphy and hepatosplenic scintigraphy were all normal.

Q Fever serology by Complement Fixation (CF) was eventually performed and confirmed by an acute infection. CF titers were $\geq 1: 2048$, (reference $\geq 1: 40$ ). The patient was discharged with a treatment of vibramycin $100 \mathrm{mg}$ twice a day for 19 days. Serologies done over the following year showed an improvement ( $\geq 1: 512$ one year later) and follow up was ended (Table 1). The chart does not mention why the follow-up was ended before negative titers $(<1: 40)$ were achieved. The patient was asymptomatic after treatment.

\section{Chronic vascular infection}

Twelve years later, in September 2010, at the age of 70, the man consulted a local primary care clinic with mild new right lower 
quadrant abdominal pain and chills. A non-infused abdominal CTscan was performed to rule out any intra-abdominal pathology such as diverticulitis. It showed a diffuse inflammation touching the anterior portion of the infrarenal aorta, which measured a diameter of $46 \mathrm{~mm}$. Possible diagnosis were, an inflammatory or mycotic aneurysm or hematoma stemming from an encapsulated rupture. There was also an asymmetry between the left and right psoas muscles. The man was afebrile with a normal complete blood count. His pain was really slight and it was chosen to complete the investigation and perform close follow-up. He was not properly interested by surgery.

An abdominal angioscan was done few days later showing the previously described inflammation on the anterior portion of the aorta, but lumen of the aorta was in fact $36 \mathrm{~mm}$ diameter (Figure 1a). However, a collection was discovered in the left psoas (Figure 1b). An aspiration of this psoas collection was CT-guided and demonstrated a whitish viscous substance not frankly purulent. Final cytological results showed necrotizing granulomatous inflammation with severe lymphoplasmacytic inflammation. There were no micro-organisms nor was there any evidence of neoplasia. Transesophageal echocardiograms did not show any signs of endocarditis. A white-blood-cell scintigraphy was also negative.

Two weeks later, the control CT scan showed signs of deterioration with a newly found ulceration of the aorta. The aortic inflammation had also progressed in size. A surgical treatment was then decided with the patient.

Resection of all inflammatory aorta and periaortic tissue adjacent to the aneurysm was performed as well as an aorto-aortic bypass with a Dacron graft. The inflammatory and granulomatous aspect of the aorta strongly suggested an infection. Autologous grafts or rifampicin drenched Dacron grafts were not available at that time in our regional centre. After completion of the debridement and graft, povidone in situ lavage of the surgical bed was performed. PCR tests on the tissue sample was later positive for Coxiella burnetii. The pathology report spoke of extensive necrosis, lymphoplasmacytic inflammation and rare granulomas.

Serologies by Immunofluorescence Assay (IFA) demonstrated a phase I IgG titer $\geq 1: 32768$ and phase II IgG titer $\geq 16384$. A long term treatment of doxycycline and hydroxychloroquine was started with a close serologic follow up on an outpatient basis. A few months later, an abdominal angiographic CT-scan showed a residual infiltration of the aorta. However, a PET-scan essentially ruled out infection of the graft.

Follow up serologies demonstrated a slight improvement immediately following the surgery but have been fluctuating every since (Table 1). The treatment has proven to be difficult because many side effects have forced the patient to cease the medication on numerous occasions. Observance to the treatment was also frequently questioned by the patient. It was stated to the patient that antibiotics are likely to be required for life due to the high risk of graft infection but he is not really compliant to serologic follow-up nor to antibiotics. Annual CT-scan was accepted by the patient and always shown stability in the images, with few residual infiltration of the aorta and no changes on the graft site (to-date, last CT was in May 2015).

In July 2015 the man was still alive and without infectious symptoms. This information was obtained on a follow-up visit with his family physician.

\section{Discussion}

Q fever is a zoonosis caused by the obligate intracellular bacterium Coxiella burnetii [1] . First described in Australia, the most recent epidemic took place in the Netherlands in 2007 [2]. Its presence is endemic in almost every country worldwide. In North America, it has most often been reported in Nova Scotia [1].
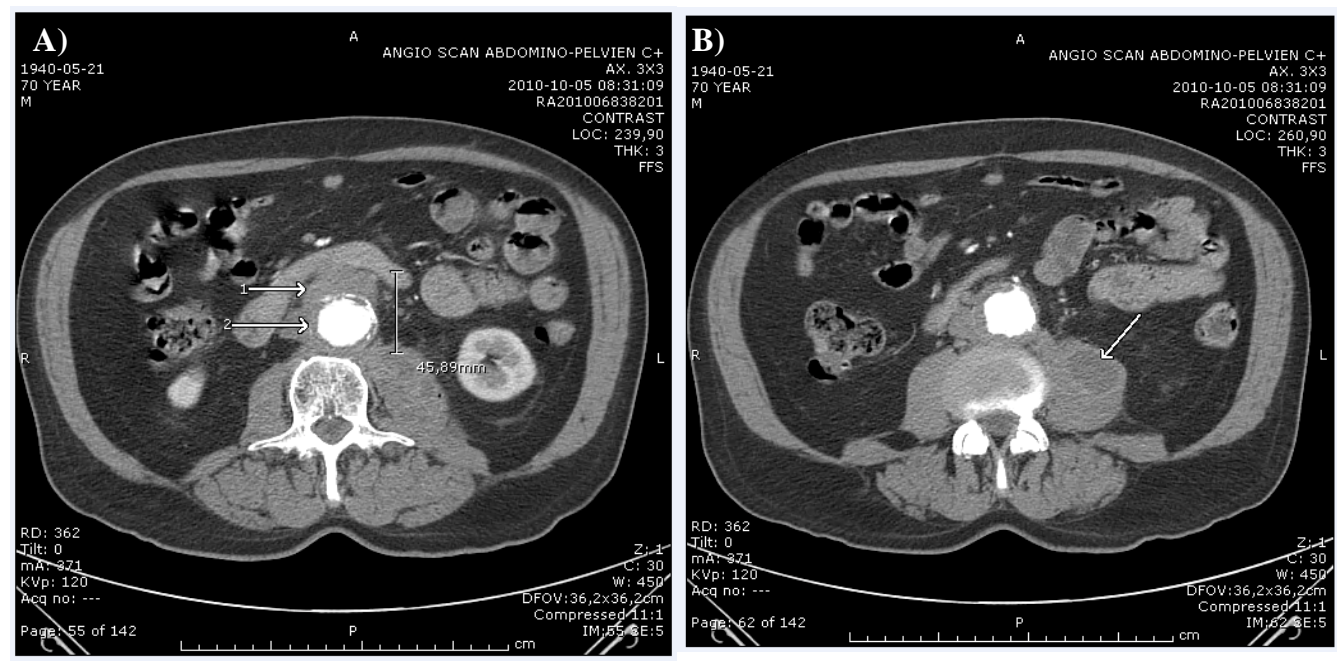

Figure 1: A) Abdominal angioscan showing an inflammatory aneurysm of the abdominal aorta, total diameter $46 \mathrm{~mm}$. There is a lot of inflammation on the anterior wall (1). The aorta lumen is measured $36 \mathrm{~mm}$ (2).

B) A collection was found in the left psoas. An aspiration with CT-SCAN guidance demonstrated a severe necrosing granulomatous inflammation. 
Table 1: Q-Fever serologic titers (not exhaustive) Serologic type.

\begin{tabular}{|l|c|c|c|}
\hline Date $^{\mathbf{a}}$ & $\mathbf{C F}^{\mathbf{b}}$ & IgG phase 1 & IgG phase 2 $^{\mathbf{c}}$ \\
\hline $12 / 11 / 1998$ & $1: 2048$ & - & - \\
\hline $2 / 19 / 1999$ & $1: 4096$ & - & - \\
\hline $11 / 11 / 1999$ & $1: 512$ & - & - \\
\hline $12 / 16 / 2010$ & $1: 128$ & $1: 32768$ & $1: 16384$ \\
\hline $1 / 13 / 2011$ & - & $1: 16384$ & $1: 8192$ \\
\hline $6 / 23 / 2011$ & - & $1: 32768$ & $1: 16384$ \\
\hline $6 / 29 / 2012$ & - & $1: 4096$ & $1: 2048$ \\
\hline $1 / 25 / 2013$ & $1: 256$ & $1: 8192$ & $1: 4096$ \\
\hline 9/11/2013 & - & $1: 4096$ & $1: 2048$ \\
\hline $\begin{array}{l}\text { a: Date format: Month-day-year } \\
\text { b: Complement fixation technique } \\
\text { c: Immunofluorescent assay technique }\end{array}$ \\
\hline
\end{tabular}

Transmission of Q fever occurs mostly through aerosols from urine, stool, milk and especially birth products of infected farm animals. Less frequent forms of transmission include ingestion of non-pasteurized dairy products, transcutaneous transmission (primarily by infected ticks) and vertical transmission. However, many affected patients live in urban areas or did not have any direct contact with animals. Wind plays an important role in carrying aerosols to this population. Finally, human-to-human transmission is exceptionally rare [1].

The manifestations of the infection can be acute, chronic as well as subclinical. The acute phase of the infection is often asymptomatic (approximately 60\%). When symptomatic, the acute disease is most often described as a febrile or flu like syndrome (17\%), hepatitis (40\%) and atypical pneumonia (17\%). Rare manifestations include central nervous system infection, pericarditis or myocarditis (1\% each). A non-specific rash (11\%) and thrombocytopenia (35\%) are possible presentations of an acute infection [1]. Because the clinical presentation of $Q$ fever is polymorphic and nonspecific, the incidence of acute $Q$ fever among humans is probably underestimated [3].

Chronic $Q$ fever is defined as an infection that persists for over 6 months. It can occur months or years after the acute episode. The chronic disease can develop in $1.5-11 \%$ of patients $[1,4]$. Previous studies showed the most common form of chronic $\mathrm{Q}$ fever, is culture-negative endocarditis (78\%) followed by vascular infections (9\%) and primarily infected aortic aneurysms. It can also be rarely found as an osteoarticular infection $(2 \%)$ or as a form of chronic hepatitis $(3 \%)[1,3,5]$.

Since 2007, the Netherlands has experienced the world's largest outbreak of Q fever, with more than 4000 notified acute $\mathrm{Q}$ fever cases [6]. Many new data were collected on the disease. In the Dutch $Q$ fever epidemic, abdominal aortic and/or iliac disease is seemingly a more significant risk factor in developing chronic $Q$ fever than a history of cardiac valve surgery and more patients was found with vascular chronic $Q$ fever compared to patients presenting with $Q$ fever endocarditis $[2,6,7]$. Hypothesis is now that acute infection evolves into a chronic infection as a continuous process.

Significant host risk factors responsible for chronicisation now include valvular surgery, vascular and cardiac prosthesis, aneurysms, renal insufficiency in age $>60$ years-old [2]. Non hematoloigc malignancy, immunosuppression and pregnancy also seemed to be risk factors, but these still remain uncertain. Further studies on the subject are needed and ongoing. Reports showed no elevated risk for patients with mild valvulopathy in the Netherlands outbreak [8].

Q-fever diagnosis is most often made with serologies. In acute infection, titers take 1 to 2 weeks to become positive and negativization occurs up to a year later. Serologic testing allows differentiation between acute and chronic $Q$ infection from the antibodies profile. Acute $\mathrm{Q}$ fever is characterized by antiphase II antibodies, whereas anti-phase I antibodies are seen in the chronic phase [3]. Phase I antibody titers that persist (typically IgG $>1: 1024$ ) for over 6 months after an acute infection, that reappear or that are initially superior than phase II antibody titers suggest a chronic infection. After an acute infection, patients should be followed both clinically and serologically $[1,5,9]$.

Until recently, recommendations concerning serological follow-up for the identification of chronic $Q$ fever following an acute $Q$ fever infection in patients with one or more risk factors were often based on clinical experience. Hagenaars et al. [7] proposed in a recent publication a standardized and differentiated serological follow-up of patients with aortoiliac disease and Q fever infection. Their approach is promising and they propose to continue the follow-up of their cohort in the future to validate it. Serological testing is done every 3,6 or 12 months, relying on the specific serological profile of the patient. In case of a negative serological profile (negative IgG phase II and negative IgG phase I), they propose the patient should be discharged for follow-up.

There is sufficient evidence to support serological followup of all known acute Q-fever patients at least once during the first year following the acute infection, and more frequently in patients with known risk factors for chronic disease [10]. Also, the targeted screening programme for risk groups should be considered in outbreaks of $Q$ fever [6].

Acute infections can be treated when diagnosis is made before resolution of symptoms. Usual treatment consists of $100 \mathrm{mg}$ of doxycycline BID for 14 days [9]. Treatment for chronic infections has mainly been studied for endocarditis and requires long-term antibiotics (more than 18 months) as well as a close follow-up of antibody titers $[9,11]$. Doxycycline is then often combined with either hydroxychloroquine, rifampin or quinolone $[3,9,12]$. When endocarditis or vascular infection is proven, treatment should be at least 3 years of double coverage [3]. Certain experts recommend a lifelong treatment [13] as there have been cases of relapse after cessation of antibiotics in spite of over 3 years of treatment [14]. Most crucial of all is to pursue treatment when antibody titers remain elevated [9].

In vascular infection, surgery is typically indicated, as it has 
been shown to significantly improve prognosis [11]. Several open surgical options exist for mycotic aortic aneurysms. These include open aortic reconstruction using femoral veins, aortic homografts or rifampin-bonded Dacron grafts with an omental pedicle flap. Endovascular reparation with rifampin soaking dacron-based endografts is also a promising option for poor operative candidates [15]. Even if there is a flourishing new literature on surgical options for treatment of mycotic aneurysm, hard data are unfortunately still lacking on the subject.

Our case-report raises few questions to be resolved by further investigations. First, it is of interest to consider that the patient did not have any aorta aneurysm at the time of is acute infection. It may be hypotheses that Coxiella burnetii caused the aneurysm on previous atherosclerosis instead of settled within a pre-existing aneurysm and causes it to grow. Evidences on the mehcanism for the development of aneurysms by Coxiella burnetii are lacking.

Second, the finding of necrotizing granulomatous infiltration in the aspiration sample of the psoas collection and also in the aortic wall is relevant. These histologic findings are well described for the aortic wall [16]. The Psoas collection distant from aneurysm site is not an usual finding. For Hagenaars et al, [17] clinicians and pathologists should be aware that Chronic Q fever should be included in the list of infectious diseases with necrotizing granulomatous response, such as tuberculosis, cat scratch disease and syphilis.

Third, a very few cases of $\mathrm{Q}$ fever describing both the acute and chronic episodes in the same patient have been described. In the case series by Raoult et al. [1], both phases were reported in only 19 of 1383 cases. Among these 19 cases, 3 had an infected aortic aneurysm. The latency between the acute and chronic episodes was between 1 and 3 years. Whereas long latencies have been described in cases of endocarditis (up to 20 years) [18], the unusually long latency between these two episodes (12 years) is, to our knowledge, the longest to have been described in a case of chronic vascular infection.

We can also mention that the clinical outcome of the patient is still good, even if a Dacron graft was installed and patient compliance to antibiotics and serologic follow-up is not strong.

\section{Conclusion}

The purpose of this case report is to increase the level of suspicion for $\mathrm{Q}$ fever even in low-incidence regions and show the importance of serological follow-up after acute infection. The disease is vastly underdiagnosed and awareness must be raised in order to recognize and properly treat an acute infection. Also, one must be aware of the risk factors for chronic infection as well as the possibility of a long latency of the chronic infection before being symptomatic.

Unfortunately, an adequate treatment of acute $Q$ fever does not preclude a chronic infection. The serological and clinical follow up of such patients are critical. In this case, the patient was lost to follow-up for twelve years before presenting with a Q-fever infected aneurysm. It is thought of importance to keep a high level of suspicion of chronic infection in anyone who reports a past acute episode. When chronic infection is diagnosed, serologic follow-up is crucial and treatment is sometimes required for life.

This case-report also presents multiple interesting topics such as the founding of necrotizing granulomatous inflammation in a distant psoas abcess and the fact that no aneurysm was present at the time of primary infection.

\section{Acknowledgment}

The researchers declare that they have no conflicts of interest. The authors wish to thank Department of Family Medicine and Emergency Medicine of Laval University for support.

\section{References}

1. Raoult D, Tissot-Dupont H, Foucault C, Gouvernet J, Fournier PE, Bernit E, et al. Q fever 1985-1998.Clinical and epidemiologic features of 1,383 infections. Medicine (Baltimore). 2000;79(2):109-23. doi: 10.1097/00005792-200003000-00005.

2. Wegdam-Blans MC, Kampschreur LM, Delsing CE, Bleeker-Rovers CP, Sprong T, van Kasteren ME, et al. Chronic Q fever: Review of the literature and a proposal of new diagnostic criteria. J Infect. 2012;64(3):247-59. doi: 10.1016/j.jinf.2011.12.014.

3. Sessa C, Vokrri L, Porcu P, Maurin M, Stahl JP, Magne JL. Abdominal aortic aneurysm and Coxiella burnetii infection: report of three cases and review of the literature. J Vasc Surg. 2005;42(1):153-8. doi:10.1016/j.jvs.2005.03.022.

4. Fournier PE, TJ Marrie, D Raoult. Diagnosis of Q fever. J. Clinical Microbiol.1998;36(7):1823-1834.

5. Raoult D, Marrie T, Mege J. Natural history and pathophysiology of Q fever. Lancet Infect Dis. 2005;5(4):219-26. doi: 10.1016/S14733099(05)70052-9.

6. Wegdam-Blans MC, Stokmans RA, Tjhie JH, Korbeeck JM, Koopmans MP, Evers SM, et al. Targeted screening as a tool for the early detection of chronic Q fever patients after a large outbreak. Eur J Clin Microbiol Infect Dis. 2013;32(3):353-9. doi: 10.1007/s10096-012-1749-9.

7. Hagenaars JC, NH Renders, AS van Petersen, SO Shamelian, MG de Jager-Leclercq, FL Moll, et al. Serological follow-up in patients with aorto-iliac disease and evidence of $\mathrm{Q}$ fever infection. European J. Clinical Microbiol. Infectious Dis.2014;33(8):1407-1414. doi: 10.1007/s10096-014-2084-0.

8. Limonard GJ, MH Nabuurs-Franssen, G Weers-Pothoff, C Wijkmans, R Besselink, AM Horrevorts, et al. One-year follow-up of patients of the ongoing Dutch Q fever outbreak: clinical, serological and echocardiographic findings. Infection.2010:38(6):471-7. doi: 10.1007/s15010-010-0052-x.

9. Anderson A, H. Bijlmer, PE. Fournier, S GravesI, J Hartzell, et al. Diagnosis and management of $Q$ fever-united states,2013: Recommendations from CDC and the $Q$ fever working group. CDC MMWR Recommendations Reports. 2013;62(3):1-30.

10. Wielders CC, Morroy G, Wever PC, Coutinho RA, Schneeberger PM, van der Hoek W. Strategies for early detection of chronic Q-Fever: a systematic review. Eur J Clin Invest. 2013;43(6):616-39. doi: 10.1111/eci.12073.

11. Botelho-Nevers E, Fournier PE, Richet H, Fenollar F, Lepidi H, Foucault $\mathrm{C}$, et al. Coxiella burnetii infection of aortic aneurysms or vascular grafts: Report of 30 new cases and evaluation of outcome. Eur J Clin 
Microbiol Infect Dis. 2007;26(9):635-40. doi: 10.1007/s10096-0070357-6.

12. Kloppenburg GT, van de Pavoordt ED, de Vries JP. Endograftpreserving therapy of a patient with Coxiella burnetii-infected abdominal aortic aneurysm: A case report. J Med Case Rep. 2011;5:565 doi: 10.1186/1752-1947-5-565.

13. Raoult D, S.B Claderwood, J. Mitty. Q fever endocarditis. 2012. Available from: http://www.uptodate.com/contents/q-fever-endocarditis

14.Wegdam-Blans MC, Vainas T, van Sambeek MR, Cuypers PW, Tjhie HT, van Straten AH, et al. Vascular complications of Q-fever infections. Eur J Vasc Endovasc Surg. 2011;42(3):384-92. doi: 10.1016/j. ejvs.2011.04.013.

15. Escobar GA, Eliason JL, Hurie J, Arya S, Rectenwald JE, Coleman DM. Rifampin soaking dacron-based endografts for implantation in infected aortic aneurysms--new application of a time-tested principle. Ann Vasc Surg. 2014;28(3):744-8. doi: 10.1016/j.avsg.2013.10.006

16. Hagenaars JC, Koning OH, van den Haak RF, Verhoeven BA, Renders $\mathrm{NH}$, Hermans $\mathrm{MH}$, et al. Histological characteristics of the abdominal aortic wall in patients with vascular chronic Q fever. Int J Exp Pathol. 2014;95(4):282-9. doi: 10.1111/iep.12086.

17.Julia C.J.P. Hagenaars, Peter CW, André S. van Petersen, PJ. Lestrade, Monique G.L. de Jager-Leclercq, et al. Estimated prevalence of chronic q fever among Coxiella burnetii seropositive patients with an abdominal aortic/iliac aneurysm or aorto-iliac reconstruction after a large dutch q fever outbreak. Journal of Infection. 2014;69(2):154160. doi: dx.doi.org/10.1016/j.jinf.2014.03.009.

18. Wilson HG, Neilson GH, Galea EG, Stafford G, O'Brien MF. Q fever endocarditis in Queensland. Circulation. 1976;53(4):680-4. 